\title{
Le comblement de la ria de Gernika (golfe de Gascogne) à l'Holocène terminal
}

\author{
Ana PASCUAL ${ }^{a *}$, Olivier WEBER ${ }^{\text {b }}$, Julio RODRIGUEZ-LAZARO ${ }^{\text {a }}$, Jean-Marie JOUANNEAU ${ }^{\text {, }}$, \\ Michel PUJOS ${ }^{\mathbf{b}}$
}

${ }^{a}$ Universidad del Pais Vasco /E.H.U. Facultad de Ciencias, Departamento de Estratigrafia y Paleontologia, Apartado 644, E-48080 Bilbao, Spain

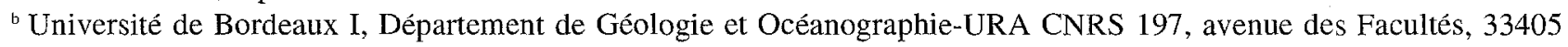
Talence-cedex, France

(Revised 29/12/97, accepted 05/01/98)

\begin{abstract}
The filling of the ria de Gernika (Bay of Biscay) since the late Holocene. A study of the recent sedimentation of the ria de Gernika (Bay of Biscay) is undertaken to characterise different steps of its Holocene filling. Sedimentological and micropalaeontological analysis of six cores sampled in the ria, completed with absolute dating $\left({ }^{14} \mathrm{C}\right.$, and based upon ${ }^{210} \mathrm{~Pb}$ exc.), allow us to identify three phases in the filling of the ria that are limited by two transgressive shifts ( 3500 and $2500 \mathrm{yr}$. B.P.) already described in other areas of the Bay of Biscay. In the first phase, lithologies as well as benthic foraminifera and ostracode assemblages are typical of coastal marine palaeo-environment. During the second phase the euryhaline waters progessively occupy the central area of the estuary. Here the presence of allochthonous microfauna, particularly of planktonic foraminifera, indicates an increase of exchanges with marine waters that can reach the inner areas of the estuarine system. The last phase ( $2500 \mathrm{yr}$. B.P. to the present), which corresponds to the recent and active sedimentation, is marked by an impoverishment of the microfaunas, the filling of some channels and the increase of salt marshes. The estimated sedimentation rate for the upper decimetres of the corcs $\left(0.73-1.29 \mathrm{~cm} \cdot \mathrm{yr}^{-1}\right)$ shows faster sedimentary filling of the ria with anthropic action evidenced by the presence of metallic contaminants and hypohaline microfauna. (C) Elsevier, Paris
\end{abstract}

\section{microfauna / sedimentation / late Holocene / ria de Gernika}

Résumé - Une étude de la sédimentation récente de la ria de Gernika (golfe de Gascogne) est entreprise dans le but de déterminer les diverses étapes de son comblement holocène. L'analyse sédimentologique et micropaléontologique de six carottes prélevées dans la ria, complétée par des datations absolues $\left({ }^{14} \mathrm{C}\right)$ et l'estimation de taux de sédimentation basées sur la mesure du ${ }^{210} \mathrm{~Pb}$ exc. permettent d'identifier trois épisodes dans le comblement de la ria qui correspondraient à deux pulsations transgressives (3500 et 2500 ans B.P.) déjà observées dans d'autres secteurs du golfe de Gascogne. Dans le premier épisode, les lithologies et les associations de foraminifères benthiques et d'ostracodes montrent un paléoenvironnement marin côtier. Dans le second épisode, les paléoenvironnements euryhalins gagnent progressivement la partie médiane de l'estuaire avec, au même moment, la présence de microfaunes allochtones (foraminifères planctoniques surtout) qui suggère une augmentation des échanges avec les eaux marines jusque dans la partie la plus amont du système. L'épisode 3 (2500 ans B.P. à l'actuel) correspond à une sédimentation récente toujours active ; elle se traduit par un appauvrissement de la microfaune, un colmatage des chenaux et une extension de la zone de schorre. Les taux de sédimentation dans les derniers décimètres $(0,73-1,29 \mathrm{~cm} / \mathrm{an})$ témoignent d'une accélération du comblement de la ria durant le dernier siècle au cours duquel l'action anthropique se manifeste par la présence de contaminants métalliques ( $\mathrm{Pb}$ et $\mathrm{As}$ ) et d'une faune hyposaline. (C) Elsevier, Paris

microfaune / sédimentation / Holocène terminal / ria de Gernika

* Correspondence and reprints 


\section{INTRODUCTION}

La ria de Gernika s'étale sur une longueur de10,6 km et sur une largeur maximale de $1 \mathrm{~km}$ dans un bassin hydrographique de près de $150 \mathrm{~km}^{2}$ (figure l). Sa profondeur

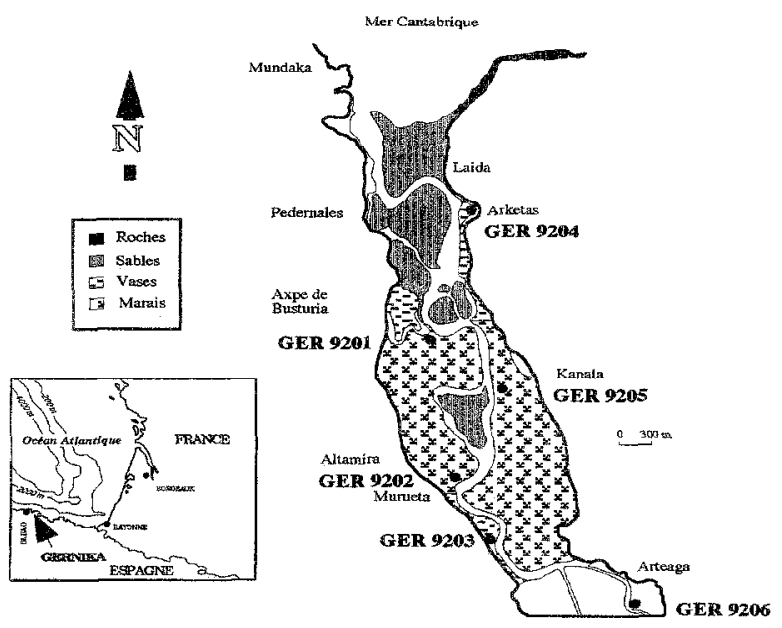

Figure 1. Carte de la ria de Gernika avec localisation des carottes étudićes.

Figure 1. Map of the ria de Gernika with location of the studied cores.

moyenne est de $2,6 \mathrm{~m}$, atteignant $4 \mathrm{~m}$ dans sa partie aval. Les biotopes marins, estuariens et fluviatiles qui se succèdent sur de faibles distances, offrent une vision intégrée d'un système particulièrement favorable à l'enregistrement et à l'étude de l'interface continent-océan.

Divers travaux ont été réalisés dans la ria concernant I'hydrographie et la sédimentologie $[5,15]$, la minéralogie et la géochimie des sédiments (Irahien, 1988), et le microbenthos $[10,11]$. Les bioconoses de foraminifères benthiques et d'ostracodes, étroitement liées à ces milieux, réagissent aux moindres variations des caractéristiques physiques et chimiques de l'environnement. L'analyse micropaléontologique des associations de la ria a permis à Pascual $[10,11]$ de différencier deux unités géographiques:

La zone amont dominée par des marais à Spartina maritima sur un substrat vaseux. Elle est caracterisée par de fortes variations de la salinité (1-32, des concentrations élevées en nutrients et une grande quantité de matière organique [5]. Les espèces dominantes sont: Ammonia tepida, Haynesina germanica et Elphidium articulatum (foraminifères) et Leptocythere castanea et Loxoconcha elliptica (ostracodes).

La zone aval, soumise à une influence marine dominante. Les valeurs de la salinité y sont plus stables (3234) et les concentrations en sels nutritifs plus faibles. Le substrat sableux occupe les plages et les bancs de la ria. L'association prédominante est composée de Cibicides lobatulus, Quinqueloculina seminulum et Elphidium crispum (foraminifères) et de Urocythereis oblonga et Aurila convexa (ostracodes).

Cette bonne connaissance de la distribution des environnements actuels permet d'aborder l'étude des paléoenvironnements de la ria. Le présent travail propose une interprétation lithostratigraphique et micropaléontologique basée sur l'étude des dépôts carottés. L'analyse des paléomilieux successifs nous permettra de mieux cerner les relations entre les paléothanatocœenoses de foraminifères et d'ostracodes, les modifications de la nature et de l'importance des apports et les variations du niveau marin, dans le but d'esquisser les grandes lignes évolutives de la ria de Gernika au cours des derniers épisodes du Quaternaire.

\section{STRATÉGIE ET MÉTHODES}

Six carottes prélevées dans l'ensemble de la ria, d'une longueur maximale de $2,20 \mathrm{~m}$, ont fait l'objet d'une étude pluridisciplinaire (figure 1). L'étude sédimentologique comprend, outre les observations à l'ouverture, des analyses radiographiques (Baltograph), microgranulométriques (difractomètre laser Malvern $2600 \mathrm{E}$ ) et les teneurs en carbonates (calcimètre Bernard). L'analyse géochimique des sédiments (composants majeurs et éléments traces) a été realisée par spectrométrie de fluorcscence $X$ sur GER 9201 et GER 9206. L'étude micropaléontologique a été effectuée sur les résidus de la fraction $>63 \mu \mathrm{m}$ après lavage à l'eau, tamisage et séchage du sédiment. trois cents foraminifères (lorsque cela était possible) et tous les ostracodes présents ont fait l'objet d'une analyse taxonomique ainsi que du calcul des indices de richesse et de diversité [12].

Des datations absolues au $14_{C}$ ont été realisées sur des tests de foraminifères, des coquilles de mollusques et des restes de matière organique par AMS (Accelerator Mass Spectrometry) au laboratoire Beta Analytic In. (Université Branch, Florida. U.S.A.). Les taux de sédimentation sont évalués par Spectrometrie gamma Haute Résolution 
de radionucléides $\left({ }^{210} \mathrm{~Pb},{ }^{137} \mathrm{Cs}\right)$ sur les carottes GER 9201, GER 9203 et GER 9206.

\section{RÉSULTATS}

Les résultats sédimentologiques des six prélèvements effectués revèlent cinq faciès granulométriques principaux auxquels sont associés certains éléments figurés (figure 2) et structures sédimentaires. Ces faciès correspondent aux différents environnements actuellement observables en surface déjà décrits par Valencia et Borja [15]:

Le faciès des sables grossiers et graviers n'est observé que sur la carotte GER 9203 vers $135 \mathrm{~cm}$ de profondeur.

Le faciès des sables moyens $(250 \mu \mathrm{m}<\varnothing<500 \mu \mathrm{m})$, identique à celui des chenaux actuels comportant des galets mous de vase, des débris coquilliers et quelques laminations horizontales, est présent à la base des carottes de la partie médiane de la ria (GER 9201 et GER 9205).

Les sables fins $(125 \mu \mathrm{m}<\emptyset<250 \mu \mathrm{m})$ constituent le faciès le mieux représenté dans la partie aval de la ria (GER 9201, 9202, 9204 et 9205); associés à des lits de débris coquilliers, ils constituent souvent des bases de séquences granodécroissantes au dessus d'un contact érosif.

Le faciès des sables très fins $(63,5 \mu \mathrm{m}<\emptyset<125 \mu \mathrm{m})$ peu représenté (GER 9204 et GER 9202), est caracterisé par des laminations subhorizontales.

Les vases $(\varnothing<63 \mu \mathrm{m})$ de couleur grise et noire, caractéristiques des zones humides actuelles, sont présentes au sommet de quelques séquences granodécroissantes des carottes de la zone médiane mais principalement dans les deux carottes situées dans le fond de la ria (GER 9203 et GER 9206) et au sommet de la GER 9201. Certains niveaux de vases noires sont riches en matière organique représentée parfois par des niveaux ligneux et témoignent de la colonisation par des végétaux dans ces zones. L'ensemble de ces observations permet d'associer d'une manière générale les prélèvements de la zone aval (GER 9204, GER 9205 et GER 9202) ainsi que la base de la GER 9201 d'une part, et les prélèvements de la zune amont (GER 9203 et GER 9206) d'autre part. Ainsi, la zonc pour laquellc le sédiment apparât le plus grossier avec une fraction sableuse très importante correspond aux carottes prélevées en bordure du chenal, là où l'influence marine est la plus forte. Au niveau sédimento- logique, ce caractère marin est perçu au travers d'un fort pourcentage de carbonates compris entre 10 et $40 \%$. A titre d'exemple, la première carotte, GER 9204, prélevée dans la partie la plus aval de la ria en bordure du chenal dans l'anse d'Arketas, est constituée principalement de sables, avec de nombreux débris coquilliers. Organisée en séquences granodécroissantes, elle révèle une forte influence de l'hydrodynamique (niveau coquillier à Turritelles -"shell lag"- au-dessus d'un contact net à la base de la séquence). Plus en amont, aux environs de Kanala, le prélèvement de la GER 9205 sur la rive droite du chenal montre encore une sédimentation grossière sableuse dans laquelle sont observés de nombreux galets de vase et plusieurs niveaux coquilliers. Là encore ces faciès témoignent d'un environnement à forte énergie capable d'éroder les vases cohésives situées dans le fond de la ria. Il est à noter que le prélèvement GER 9201, quoique situé à proximité du chenal, montre que ce secteur particulier est en voie de comblement. En effet si, depuis la base de la carotte $(209 \mathrm{~cm})$ jusqu'à $130 \mathrm{~cm}$ de profondeur, elle présente un faciès grossier comparable aux 3 précèdentes carottes, le reste de la colonne sédimentaire (de $130 \mathrm{~cm}$ au sommet) montre une sédimentation beaucoup plus fine et des teneurs en carbonates extrèmement faibles $(<1,5 \%)$. Les faciès vaseux de la partie amont correspondent principalement aux vases observées dans les carutles GER 9203 et 9206 dans lesquelles les teneurs de carbonates n'cucèdent pas $5 \%$. L'étude micropaléontologique qui a permis d'identifier 77 espèces de foraminifères benthiques et 36 espèces d'ostracodes a fait l'objet d'un précé dent travail (Pascual et Rodriguez-Lazaro, 1996). L'analyse détaillée de ces microfaunes montre l'alternance d'épisodes estuariens et/ou marins, définis de manière harmonieuse par les deux groupes.

\section{DISCUSSION}

\subsection{Succession des paléomilieux}

Dans les carottes étudiées (figure 2), deux types de paléoenvironnement ont été reconnus, marin et estuarien. Les modifications des pourcentages des types d'association de foraminifères et d'ostracodes et les observations des transitions latérales de faciès permettent de distinguer trois épisodes dans la sédimentation :

- l'épisode 1 est caractérisé dans la partie médiane de la ria (GER 9201, GER 9202, GER 9205), par un biotope marin côtier associé à des faciès de sables moyens à gros- 


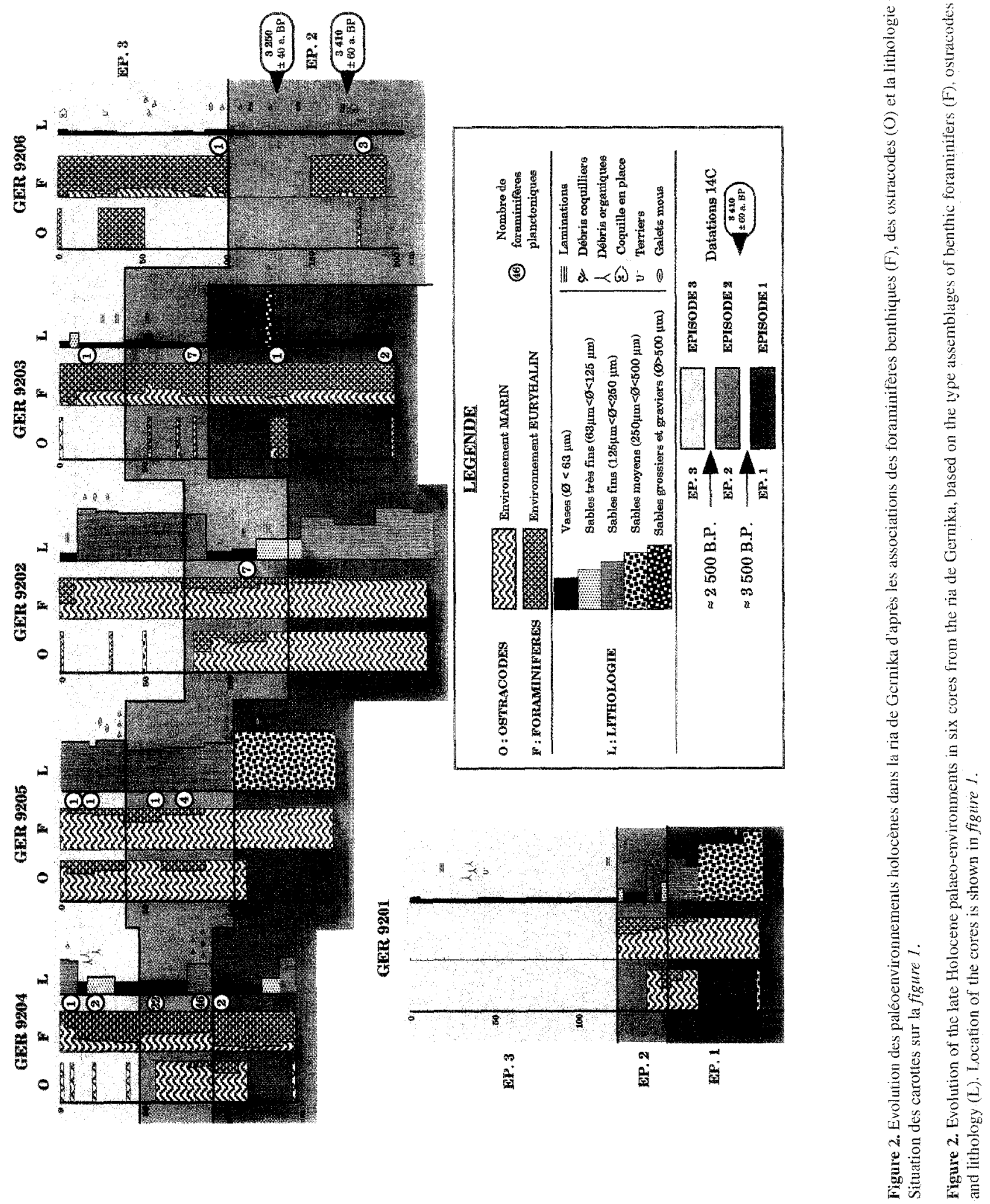


siers carbonatés $(20 \%)$. Les associations fauniques sont les suivantes : Cibicides lobatulus, Elphidium crispum, Quinqueloculina seminula, Pontocythere elongata, Aurila convexa, Leptocythere psammophila et Urocythereis oblonga. De nombreux tests, pourtant robustes, sont brisés, témoignage d'une hydrodynamique importante que l'on trouve de nos jours dans les chenaux d'estuaires à forte énergie [8].

En amont de Murueta (GER 9203 et GER 9206), dans des faciès silto-argileux, se développe sur la rive gauche un biotope euryhalin qui disparait plus en amont sur la rive droite (Arteaga).

Cette microfaune présente dans la GER 9203 comporte : Ammonia tepida, Haynesina germanica, Elphidim articulatum, Jadammina macrescens, Leptocythere castanea et Loxoconcha elliptica. Elle est comparable à celle rencontrée dans les marais supratidaux actuels de la ria. Cet épisode fait défaut dans la coupe GER 9206.

Près de l'embouchure (Arketas), une séquence granodécroissante peu carbonatée, passant de sables moyens à des vases, est liée à l'extension d'un biotope euryhalin $(90 \%)$. La GER 9204 située près d'un ruisseau montre la présence d'Ammonia tepida, Haynesina germanica, Loxoconcha elliptica, Leptocythere psammophila et L. pellucida ceci signale l'existence d'un milieu euryhalin protégé $[8,1]$.

- l'épisode 2 est caractérisé dans l'ensemble de la ria par une sédimentation plus fine souvent associée à des séquences granodécroissantes traduisant une diminution d'énergie du milieu et par la présence de formes allochtones de foraminifères planctoniques, benthiques et ostracodes [12].

J e caractère marin de la partie médiane est toujours dominant avec cependant une composante euryhaline plus marquée au sommet de l'épisode (de 20 à $40 \%$ de la population) par la présence d'Ammonia tepida, Haynesina germanica y Loxoconcha elliptica. Des tests de foraminifères planctoniques allochtones sont également rencontrés dans ce milieu où les interactions entre eaux saumâtres et marines sont plus grandes.

En amont de Murueta le caractère euryhalin dominant est accentué (jusqu'à $80 \%$ de la population).

A l'aval de la ria (Arketas), le caractère marin augmente sensiblement par rapport à l'épisode précédent $(50 \%)$. La diversité spécifique augmente également ; aux espèces euryhalines communes s'ajoutent Sarsicytheridea bradii, Eucytherura sp. et des foraminifères planctoniques représentant une part minoritaire mais toujours significative.
- l'épisode 3 présente des faciès variables traduisant les conditions de sédimentation actuelles (chenal, schorre). Les biotopes y sont variés.

Dans la partie médiane (en bord de chenal) les carottes GER 9202 et 9205 sont les plus marines.

Les caractéristiques micropaléontologiques sont variées avec une microfaune comparable à celle de l'épisode 2, caractéristique d'un milieu de chenal à forte énergie (GER 9205) : rares Jadammina macrescens typiques de chenaux de shorre (GER 9202) ; absence de faune peut être due au comblement du chenal (GER 9201).

A l'opposé, en amont, le caractère euryhalin domine. Ainsi Jadammina macrescens (50\% de la microfaune) dans la carotte GER 9203 éloignée de l'axe du chenal, indique la présence de milieux supratidaux à faible salinité et forte turbidité [13] alors que dans la carotte GER 9206 à $30 \mathrm{~cm}$, Cyprideis torosa indiquerait l'existence d'un bref intervalle hyposalin [9]. De la base des ces épisodes 2 et 3 jusqu'à $10 \mathrm{~cm}$ du toit des coupes, des thanatocœnoses de transport (Cibicides lobatulus, foraminifères planctoniques) marquent le maintien d'une ouverture océanique sur la ria et la permanence de l'action de la marée jusque dans le fond de la ria.

Dans la partie aval (GER 9204) la composante euryhaline toujours dominante, diminue vers le sommet (de 70 a $40 \%)$.

\subsection{Histoire du comblement de la ria}

Afin de disposer de repères chronologiques dans les trois épisodes précédemment définis, trois datations absolues ont été réalisées. Dans le secteur amont (GER 9206), le niveau $170 \mathrm{~cm}$ donne un âge de $3410 \pm 60$ ans $B P$ et le niveau $130 \mathrm{~cm}$ un âge de $3250 \pm 40$ ans $B P$. Dans le domaine aval (GER 9204), la datation effectuée à $100 \mathrm{~cm}$ a livré un âge de $3740 \pm 60$ ans BP. Les limites de ces trois épisodes pourraient correspondre au maximum de deux pulsations transgressives centrées à 3500 et 2500 ans $[4,14]$ qui a permis à Cearreta (1992) d'expliquer la présence de plusieurs associations de foraminifères dans les estuaires de la Bidassoa et du rio Nervion.

Les enregistrements sédimentaires de toutes les coupes étudiées ne concernent donc que la fin de la période Holocène (subboréale et subatlantique) durant laquelle le niveau marin était proche de l'actuel.

Lors de l'épisode 1 (de la base à 3500 ans BP), la ria constitue un environnement côtier peu profond où les influences marines se font sentir en amont jusqu'à 
Murueta. Le fond de la ria est le siège d'une forte sédimentation en milieu euryhalin. Seul le prélèvement le plus aval (GER 9204) témoigne d'une sédimentation calme en milieu plus confiné à l'écart du chenal principal.

Durant l'épisode 2 (entre 3500 et 2500 ans BP) le comblement se poursuit: la ria est le lieu d'une sédimentation plus fine où le milieu estuarien gagne progressivement la partie médiane comme en témoigne le développement significatif des faunes euryhalines. Durant cette période, la présence généralisée de faunes allochtones marines absentes dans l'épisode précédent, suggère une augmentation des échanges jusque dans la partie la plus amont du système.

Depuis le début de l'épisode 3 (de 2500 ans BP à l'Actuel), la sédimentation et la morphologie de la ria sont celles qui prévalent actuellement. Bien que la partie médiane (GER 9202, GER 9205) présente un caractère marin plus prononcé, le comblement maximum se manifeste principalement par l'extension des zones humides (GER 9201, GER 9203, GER 9206).

I es données historiques montrent que la déforestation du $\mathrm{XIII}^{\mathrm{e}}$ au $\mathrm{XV}^{\mathrm{e}}$ siècle pourrait être la cause d'une plus grande érosion continentale et donc du comblement de la [5]. L'activité économique (pêche, industrie du bois, fer...) impliquait des transports maritimes à l'interieur de la ria suggérant une meilleure navigabilité dans les chenaux [3]. Depuis le XVIII ${ }^{\mathrm{e}}$ siècle, l'action anthropique se traduit par une colonisation des marais avec poldérisation de 500 ha [7].

Dans les zones humides où l'épisode 3 apparaît le plus developpé, nous avons entrepris l'étude détaillée de la sédimentation récente.

\subsection{Sédimentation récente (dernier siècle)}

Les taux de sédimentation calculés à partir de la décroissance du ${ }^{210} \mathrm{~Pb}$ dans les carottes GER 9201, GER 920.3 et GER 9206 sont de $0,96,0,73$ et $1,29 \mathrm{~cm} / \mathrm{an}$. De telles valeurs nous ont conduit à rechercher dans les premiers décimètres du dépôt des marqueurs éventuels de l'activité anthropique. Ainsi ont été analysés les deux métaux lourds suivants: $\mathrm{Pb}$ et As. Les valeurs ont été normalisées à Al203 tant à l'aval (GER 9201) qu'à l'amont (GER 9206). Si l'on applique le taux de sédimentation calculé, (figure 3) les valeurs très basses observées vers $45 \mathrm{~cm}$ correspondraient aux années 1940. A l'opposé à $25 \mathrm{~cm}$, le maximum correspondrait aux années 1970. Au sommet vers $12 / 15 \mathrm{~cm}$ où on se situerait vers 1980 , les valeurs décroissent. Ces résultats peuvent être mis en parallèle avec le début en 1943 de l'activité industrielle des chantiers navals de Murueta [5] dont le déclin commence vers les années 1980. Un autre marqueur de cette activité anthropique est la présence remarquable de faune hyposaline dans le prélèvement le plus amont (GER 9206) consécutif à un apport d'eau douce supplémentaire dû à la construction en $1923 \mathrm{~d}$ 'un canal de $5 \mathrm{~km}$ de longueur entre Gernika et Murueta [5].

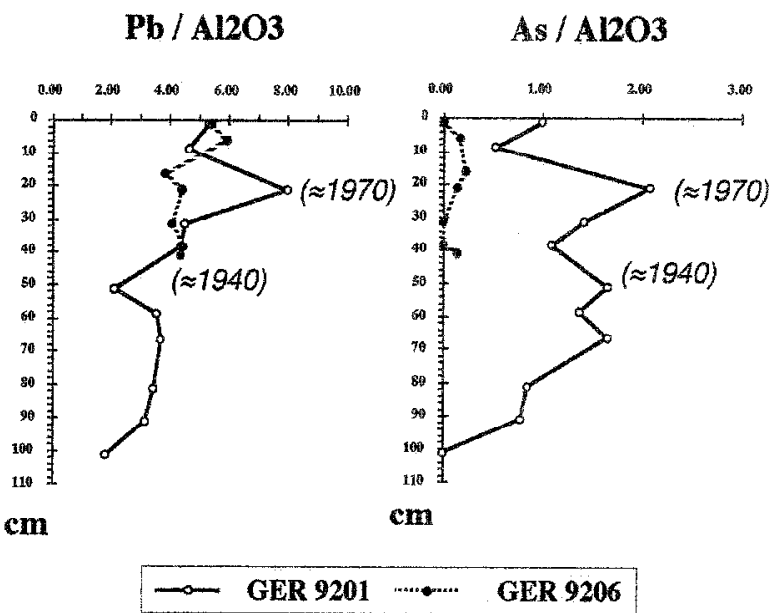

Figure 3. Distribution verticale de $\mathrm{Pb}$ et de $\mathrm{As}$ (normalisés $\mathrm{Al}_{2} \mathrm{O}_{3}$ ) dans la carotte GER 9201 de la zone médiane et la carotte GER 9206 de la zone amont de l'estuaire.

Figure 3. Vertical distribution of $\mathrm{Pb}$ and $\mathrm{As}$ (normalised to $\mathrm{Al}_{2} \mathrm{O}_{3}$ ) in the core GER 9201 in the middle part of the estuary and in the core GER 9206 in the upstream part of the ria.

\section{CONCLUSIONS}

Il ressort de cette étude les points essentiels suivants :

L'étude des microfaunes de foraminifêres et d'ostracodes permet de suivre l'évolution quaternaire récente de la ria de Gernika. Le comblement de la ria s'est effectué en trois épisodes successifs limités par deux pulsations transgressives.

Dans ce système les environnements marins et estuariens se juxtaposent :

a. l'épisode 1 supérieur à 3500 ans BP à dominante marine côtière ;

b. l'épisode 2 entre 3500 et 2500 ans BP au caractère estuarien plus affirmé ; 
c. l'épisode 3 de 2500 ans BP à l'actuel avec la mise en place progressive des biotopes actuels dans un contexte de comblement accéléré par l'homme.

La sédimentation du dernier siècle se manifeste, au travers de contaminants métalliques, par une activité anthropique importante jusqu'aux années 1980.

\section{Remerciements}

Ce travail fait partie du projet "Evolution des paléoenvironments de la ria de Gernika au cours du Quaternaire terminal" financé par le fond de Coopération Aquitaine Euskadi (1994-95).

\section{RÉFÉRENCES}

[1] Athersuch J., Horne D.J., Whittaker J.E., Marine and Brackish Water Ostracods, in: Kermack D.M., Barnes R.S.K. (Eds.), Synopsis of the British Fauna (New Series), The Linnean Society of London, Brill E.J., 43 (1989) 343 p.

[2] Cearreta A. Ecoestratigrafia (foraminiferos bcntonicos) del relleno estuarino Holoceno en el litoral vasco (Golfo de Bizkaia), Riv. Ital. Paleontol. Stratigr. 98 (1992) 243-266.

[3] Ciriquiain-Gaiztarro M., Los puertos maritimos vascongados, Ed. Txertoa, San Sebastian, 1986, 270 p.

[4] Fairbridge R.W., Eustatic changes in sea-level, in: Ahrens L.H. et al. (Eds.), Physics and Chemistry of the Earth, Pergamon Press, 4 (1961) 99-187.

[5] Gobierno Vasco Estudio oceanografico de la ria de Mundaka, Colec. Itsaso. Ser. Central Public. Gobierno Vasco, 3 (1986) 205 p.

[5] Gobierno Vasco Urdaibai reserva de la Biosfera, Ser. Central Public. Gobierno Vasco (1993) 179 p.

[6] Irahien M.I., (1993). Mineralogia geoquimica de los sédimentos actuales de los rios Nervion-Ibaizabal, Oka, Butron y Nive. Indices de gestion ambiental, Tesis Doctoral. Universidad del Pais Vasco / EHU.

[7] Meaza G., Ugarte F.M., La transformacion del espacio natural por el agrosistema vascucantabrico. La ria de Gernika-Mundaka, Lurralde 11 (1988) 137-147.

[8] Murray J.W., Ecology and palaeoecology of benthic foraminifera, Longman Scientific Technical, 1991, 397 p.
[9] Neale J.W., Ostracods and paleosalinity reconstruction, in: de Deckker P., Colin J.-P., Peypouquet J.P. (Eds.), Ostracoda in the Earth Sciences, Elsevier, 1988, 125-155.

[10] Pascual A., Ostracodes in Biscayan estuaries (Spain): their relationship with the environment, Cah. Micropal. 6 (2) (1991) 5-16.

[11] Pascual A., Utilizacion de los foraminiferos bentonicos para un mejor conocimiento del medio ambiente en los estuarios vizcainos (Gernika y Bilbao), Rev. Espanola Micropal. XXIV (1) (1992) 33-57.

[12] Pascual A., Rodriguez-Lazaro J., Micropaleontologia en materiales holocenos de la Ria de Gernika (Golfo de Vizcaya), Geogaceta 20 (1) (1996) 209-212.

[13] Pujos M., Iadammina polystoma, témoin d'un environnrment contraignant dans l'estuaire de La Gironde (France), in: Oertli H. (éd.), Benthos'83, Second Intern. Symp. on Benthic Foraminifera, 1983, 51$\rfloor-517$.

[14] Ters M., Les variations de niveau marin depuis 10000 ans le long du littoral Atlantique français, in: Le Quatcrnairc: Gćodynamique, Stratigraphie et Environnement, Travaux Récents, $9^{e}$ Congrès Intern. de 1'INQUA, Christchurch, New Zealand, 1973, 114-135.

[15] Valencia V., Borja A., Resultados de los estudios hidrograficos y sedimentologicos de AZTI-SIO en la ria de Mundaka, in: Urdaibai reserva de la Biosfera. Investigacion Basca y aplicada, Leioa. Universidad del Pais Vasco, 1993,4² Ponencia, $12 \mathrm{p}$. 